\title{
PROFESSOR J. MARKWART
}

\section{(Extract from The Times)}

THE death, in tragic circumstances, of Professor Josef Markwart, 1 of Berlin, on 4th February, ought not to pass unnoticed in this country. For although his name is here unfamiliar to all but a small group of scholars, he occupies, and must always occupy, a place in the forefront of European Orientalists, and it would be difficult to name any past or present scholar with a wider range of learning or a more brilliant critical faculty, whether as philologist, historian, or ethnologist. He was a fine classical scholar; he could read nearly every literary language of Asia, and he had a profound knowledge of African ethnology. Though his total output of published work was comparatively small-amounting only to four or five books and a number of articles in learned journals - it may be said that every sentence he wrote bore the hall-mark of his immense learning and his rare analytical power.

Born in 1864, he began his academic career in Tübingen, where he remained till 1902, when he was appointed curator of the Ethnographical Museum and later professor in the University of Leyden. In 1912 he came to Berlin as professor of Iranian and Armenian philology, a post which he was still holding at the time of his death. Indeed, he had delivered a lecture on the day preceding this tragic event. In 1913 he published a Catalogue of the Benin Collections in the Leyden Museum, a massive volume in large quarto containing a prolegomena extending over 367 pages on the history of the trade routes and the movements of populations in North Africa from the earliest times. In the course of this great work Markwart found occasion to reveal almost every sphere of his encyclopædic learning. In his Osteuropäische und ostasiatische Streifzüge (1903), dealing with the ethnology, topography, and history of the ninth and tenth centuries, he set at rest a hundred problems and exploded as many hitherto accepted theories. But it is in the field of Turkology that he rendered the most important services. The pre-Ottoman history of the Turks still remains to be written; it is still shrouded in a mist of confusing names ; but it was Markwart who came nearest to making order out of the confusing mass of information contained in the Arabic, Persian, and Byzantine chronicles and the early Turkish inscriptions and documents. 
Markwart, during his industrious life, had accumulated a mass of notes on important subjects which he never found time to publish, and it can only be hoped that these documents will not be allowed to disappear with him.--EDIToR.

\section{A List of Professor Markwart's Works}

Assyriaka bei Ktesias: Philologus, Supplementband vi, 2, Göttingen, 1891-3, pp. 501-658.

(i) Die Vorlage von Diodor, $\beta$ 1-34.

(ii) Die echtktesianische Bestandtheile bei Diodor und in andern Fragmenten.

(ii) Die Quellen des Ktesias und die Art ihrer Benutzung.

Exkursus-

(1) Zur Seite 507.

(2) Die Chronol. der Inschrift von Behistān.

(3) Die griechische Wiedergabe der persischen $a u, w a$ und wi.

(4) Die ursprüngliche Heimat der Perser.

(5) Eine Assyrisch-babylonische Königsliste bei Ja'qūbì und Mas'ū ūī .

[This work, the first two parts of which were written in 1889 and the third in 1891, was presented in February, 1893, as thesis for the doctorate of the University of Tübingen.]

Beiträge z. Geschichte und Sage v. Ërīn: ZDMG. xlix, 1896, pp. 628-73:-

Ërān.

Pahlau.

Hyrcani-Wirk', Iberen.

Ariš.

Tiridates u. Spandijāt.

Artobanos und K. Khosrau.

Gotarzes I und Orodes I.

Die Liste d. ērān. und arm. Arsakiden.

Buzurg Kūšān-Š̃̌̌h.

Der Stammbaum d. Būyiden.

Bāu.

Die Suffixe $\check{c}, \check{z}, \check{c} \bar{i}, z \bar{\imath}$.

Enclyt. $-a n=a w . n \bar{o}$. 
Altper. franah $=$ farr.

Neuper. $\bar{z} z a \delta=$ jazata.

Inschriften d. Artax. II v. Susa und Hamadān.

Fundamente israelitischer und jüdischer Geschichte, Leipzig, 1896, gr. $8^{\circ}, 88 \mathrm{pp}$.

Historische Glossen zu den alttürkischen Inschriften: WZKM. 1898, xii, pp. 157-200.

Chronologische Untersuchungen, Leipzig, 1900, 86 pp. (=Philologus, Supp.-Band vii, pp. 637-720):--

Berossos und die babylonischen Königslisten.

Zur Chronologie von Hyksos.

Die Exodusberichte des Manetho.

Die XVIII und XIX Dynastie nach Manetho.

Die Chronologie der Aethiopen und Saiten.

Untersuchungen zur Gesch. v. Ërān, i, Göttingen, 1896, 72 pp. (=Philologus, Bd. 54, 489-527 ; Bd. 55, 212-240):-

Diodors Nachrichten über das pont. und kappad. Fürstenhaus.

Das Verhältniss der Trogus z. Diodor.

Die angeblichen Zariadrosmünzen und die Fürsten v. Sophene.

Zur Assyrischen u. Medischen Königsliste bei Ktesias.

Zur Kritik des Faustos v. Byzanz.

Hazārapet.

Der altpersische Kalender.

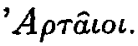

Erymandrus.

Haraiwa.

Nachträge.

$\bar{E}$ rānšahr nach der Geographie des $p_{s}$. Moses Xorenac'i, Berlin, 1901 (Abh. Gesell. Wiss. zu Göttingen, Phil.-Hist. Klasse, Neue Folge, Band ii, No, 2), $358 \mathrm{pp}$.

Erster Teil: Das Provinzverzeichniss.

Zweiter Teil : Länderbeschreibung nach Ptolemaios.

Exkurs :-

(i) Die armenischen Markgrafen.

(ii) Z. hist. Topographie v. Kermãn und Mukrān.

(iii) Toxāristān : (1) Tocharer und Ta-hia ; (2) Toxāristān unter den Wei und T'ang; (3) Toxāristān in der Steuerliste des 'Abdallāh b. Tăhir; (4) Ober- und Unter-Toxāristān; (5) Itinerar von Balx nach Ober- 


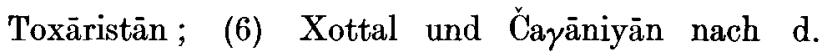
Itineraren Ișțaxrī's ; (7) Itinerare in Transoxiana ; (8) Das Gebiet v. Balx südlich v. Oxus nach Ja'qūbī;

(9) Die fünf hih-hóu Provinzen der Jüeh-či ; (10) Kābul ; (11) Das Gebiet nördlich v. Oxus nach Ja'qūbì.

Osteuropäische und Ostasiatische Streifzïge, Leipzig, 1903, 557 pp. Belaweža-Itil.

Die Bekehrung der Chazaren zum Judentem.

Die ältesten Berichte über d. Magyaren.

Der Raubzug d. Magyaren gegen Konstantinopel im Jahre 934.

Das Itinerar des Mis'ar b. Muhalhil nach d. chinesischen Hauptstadt.

Mas'ūdì's Bericht über die Slawen.

Analyse d. Berichte des Gaihāni über die Nordländer.

D. Reisebericht des Hārūn b. Jahjā.

Exkurs :-

(i) Zur Bekehrungsgeschichte d. Chazaren.

(ii) Der Stammbaum d. Abdoritenfürsten

(iii) Mas'ūdī's Bericht über die Russen und d. Ursprung des Namen Rōs.

(iv) Der Ursprung d. iberischen Bagratiden. ${ }^{1}$

(v) Ǧaihānīs Bericht über die Slawen.

Untersuchungen z. Geschichte v. Ērān, ii, Leipzig, 1905, 258 pp. (=Philologus, Supplementband x, Heft 1):-

Die Namen d. Magier.

Alexanders Marsch v. Persepolis nach Herat.

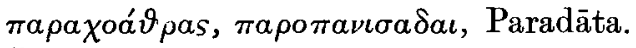

Über einige skythisch-iranische Völkersnamen.

Über einige Inschr. aus Kappadokien.

Die Chronol. d. Kambyses u. d. Lügenkönige und d. alt-pers. Kalender.

Zusätze.

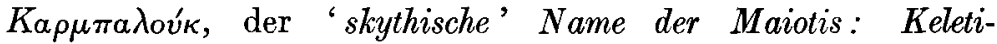
Szemle, xi, 1910, pp. 1-26.

Die altbulgarischen Ausdrücke in d. Inschrift v. Catalar und in der altbulgarischen Fürstenliste: Izv. Russ. Arch. Inst. $v$ Konstantinopolë, $\mathrm{xv}, 1911$, pp. 1-30.

1 This " Exkurs" (with some additions) was translated into Armenian by Hapozian, Handes Amsorya, 1912, 333-9, 519-31, 712-30 ; 1913, 160-7, 210-21, 281-93, 463-75. 
Armenische Streifen. (1) Historische Data z. Chronologie der Vokalgesetze ; (2) Nachtrag z. Ērānšahr : Sahastaninoknoy und zur Liste der Provinzen v. Chorāsān. Huschardzan (Festschr. d. Mechitarlsten-Congregation), Wien, 1911, pp. 291-302.

Über d. Armenische Alphabet in Verbindung mit d. Biogr.d.h. Mašt'oc: Handes Amsorya, 1911, 530-43, 673-83 (German); 1912, 41-54, 199-216 (German and Armenian) ; 657-66 (Armenian) ; 742-50 (German).

Die Benin-Sammlung des Reichsmuseums für Völkerkunde in Leiden. Beschrieben und mit ausführ. Prologomena z. Gesch. der Handelswege und Völkerbewegungen in Nordafrika versehen, Leiden (Brill), 1913, folio, ccclxvii + 132 pp. (Veröff. d. Reichsmuseums f. Völkerk. in Leiden, ii, 7).

Ğuuaini's Bericht über d. Bekehrung der Uiguren: $S B B A$. 1912, i, Halbjahr, pp. 486-502.

Südarmenien $u$. die Tigrisquellen nach griechischen und arabischen. Geographen : Handes Amsorya (German and Armenian), 1913, 79-100, $357-66,525-35 ; 1914,41-52,106-17,177-83$; 1915, 126-35; 1920, 103-10 (German).

Herkunft und Name d. Russen: Baltische Monatschr., B. 76, Heft 10, Riga, Oct. 1913, pp. 264-77.

Skizzen z. geschichtl. Völkerkunde $v$. Mittelasien und Sibirien : Ostas. Zeitschr., Jahrg. viii, 1912-20, pp. 289-99.

Über das Volkstum der Komanen, Osttürkische Dialectstudien: Abh. Gesell. d. Wissensch. zu Göttingen, N.F., Band xiii, No. 1, Berlin, 1914, pp. 25-238. ${ }^{1}$

§ 1. Uzen u. Komanen.

$\S 2$. Komanen u. Qun.

§3. Komanen u. Qypčaq.

$\S 4$. Tatar u. But-kat.
§5. Tatar u. Kimäk.

§6. Kimäk u. Qypčak.

§ 7. Qypčaq u. Komanen.

§. Qangly, Kimäk u. Qypčaq.

Anhang :-

(1) Kritik d. bisherigen Erklärungsversuche d. Namen "Falben" u. "Komanen".

(2) Über die Herkunft d. Osmanen.

(3) [Der Zug. d. Chytai nach Westen.]

${ }^{1}$ See two important reviews of this work: P. Pelliot, A propos des Comans (J.As. 1920, avr.-juin, pp. 125-85), and Barthold, Novyi trud o Polovtsax Russ, istoric. žrnal, 1921, tome 7, pp. 138-56). 
(4) Die Bedeutung d. historischen Topographie f. d. Textkritik des Schāh-nāma.

Nachtrag. [D. Chronologie d. Qara-Qytai.]

[- und GRoot], Das Reich Zābul und der Gott Žün : Festschrift f. E. Sachau, Berlin, 1915, pp. 243-92.

Mipherqēt und Tigranokerta: Handes Amsorya, 1916, pp. 68-135. pp. 68-135.

Die Entstehung und die. Wiederherstellung der armenischen Nation, Potsdam, 1919.1

Was bedeutet der Name Kaukasus?: Morgenland, No. 1, 1922, pp. 3-8.

Ein arabischer Bericht über $d$. arktischen (uralischen) Länder aus d. 10 Jahrhundert: Ungar. Jahrbücher, Berlin, iv, Heft 3-4, Dezember; 1924, pp. 261-334. ${ }^{2}$

Np. āð̄na "Freitag" : Festgabe J. Szinnyei, Ungar. Bibliothek, No. 13, Berlin, 1927, pp. 57-89.

Le berceau des Arméniens: Revue des études arméniennes, Paris, 1928, tome viii, fasc. 2, pp. 210-45.

Works announced but not yet published:--

Geschichte und historische Ethnographie des Daghestan (cf. Ëränšahr, p. 95, note 1).

: Wehrōt und Arang (cf. Über d. Volkstum d. Komanen, p. 38, note 6)of this work 160 pages have been printed by Brill (Leyden).

\section{ADDENDA.}

Die Chronologie d. alttürkischen Inschriften, Leipzig, 1898, 8vo, 112 pp. Exkurs 1. Sogdiana; Exkurs 2. D. Bulgar. Fürstenliste. Anhang: [Kök-türkisch].

Skizzen z. histor. Topographie u. Geschichte von Kaukasus. Der Itinerar von Artaxata nach Armastica auf d. römischen Weltkarte, Handes Amsorya, 1927, No. 11, pp. 825-66.

Kultur- und sprachgeschichtliche A nalekten, Ungarische Jahrbiucher, IX/1, April, 1929, pp. 68-103.

See also a list of eight articles by Professor Markwart in Handes Amsorya, 1927, p. 983, and his contributions to Amedroz, "Notes on two articles on Mayyāfāriquñ," JRAS. 1890, pp. 170-6, and Herzfeld, “Am Tor von Asien,” Berlin, 1920, pp. 39, 155 , and 180 .

The foregoing is only a provisional list of Professor Markwart's work, offered as a token of high admiration of the departed scholar's genius. I hope to re-edit it soon in a more complete form.

\section{Y. Minorsky.}

1 Abridged translation by Marie Basmadjian, L'origine et la reconstitution de la nation arménienne, Paris, $1919,8^{\circ}, 26 \mathrm{pp}$.

2 Beginning with this publication Professor Marquart changed the orthography of his name to Markwart. 